Document downloaded from:

http://hdl.handle.net/10251/100062

This paper must be cited as:

Soliveres, E.; Poveda Martinez, P.; Estruch, VD.; Pérez Arjona, I.; Puig Pons, V.; OrdoñezCebrian, P.; Ramis Soriano, J.... (2017). Monitoring fish weight using pulse-echo waveform metrics. Aquacultural Engineering. 77:125-131. doi:10.1016/j.aquaeng.2017.04.002

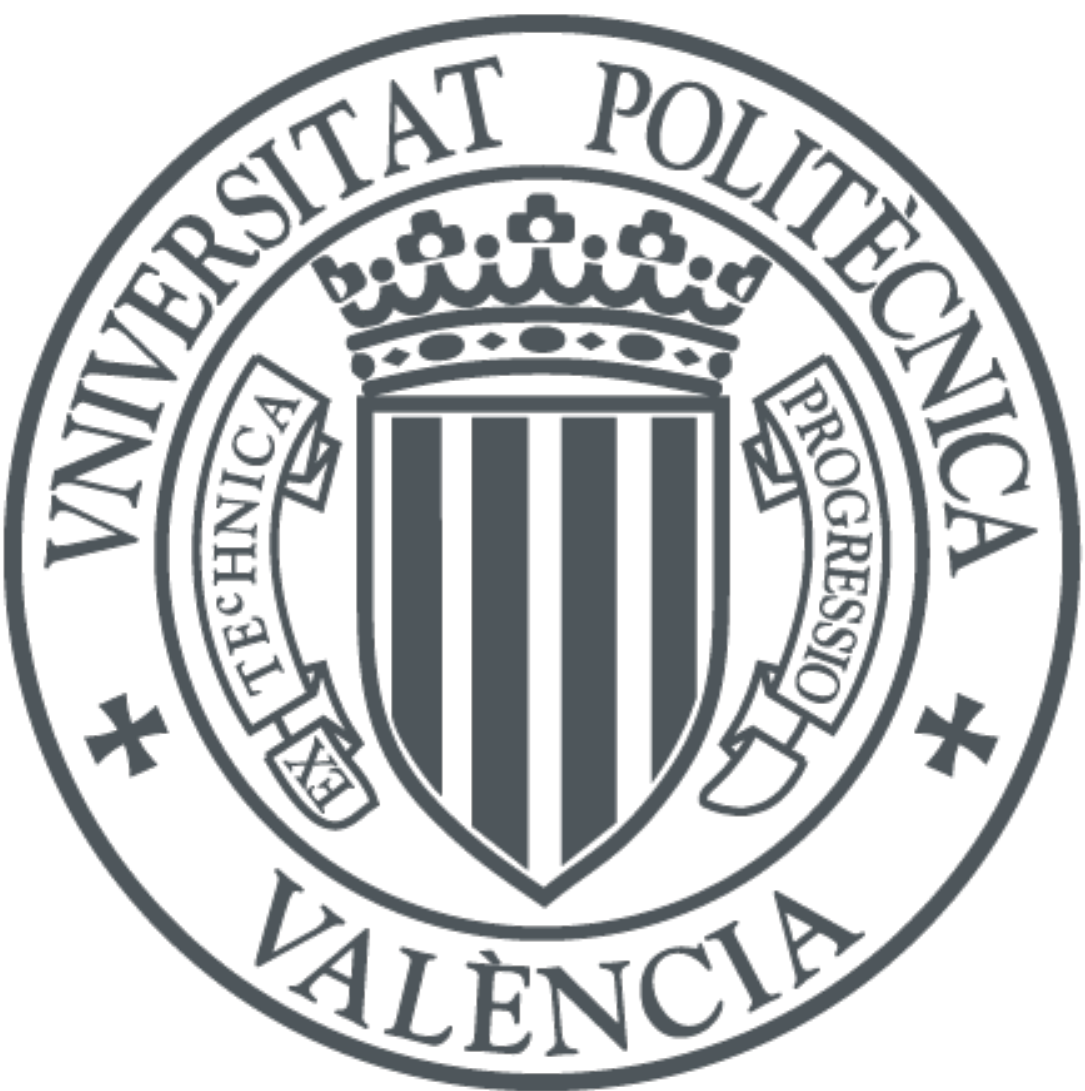

The final publication is available at

https://doi.org/10.1016/j.aquaeng.2017.04.002

Copyright Elsevier

Additional Information 


\title{
Monitoring fish weight using pulse-echo waveform metrics
}

\author{
E. Soliveres ${ }^{\mathrm{a}}$, P. Poveda ${ }^{\mathrm{b}}$, V. D. Estruch ${ }^{\mathrm{a}}$, I. Pérez-Arjona ${ }^{\mathrm{a}}$, V. Puig ${ }^{\mathrm{a}}$, P. \\ Ordóñez $^{\mathrm{a}}$, J. Ramis ${ }^{\mathrm{b}}$, V. Espinosa ${ }^{\mathrm{a}, *}$ \\ ${ }^{a}$ Institut d'Investigació per a la Gestió Integrada de Zones Costaneres, Universitat \\ Politècnica de València, C/ Paranimf 1, 46730 Grau de Gandia, Spain \\ ${ }^{b}$ Física Aplicada a les Ciències $i$ les Tecnologies, Universitat d' Alacant, Ap. de correus 99, \\ 03080, Alacant, Spain
}

\begin{abstract}
Fish anatomical vertical dimensions are extracted from a time-of-flight analysis of fish echo shape using narrow-bandwidth echosounding of swimming individuals. These vertical dimensions fit a Gumbel distribution model and are successfully correlated with fish weight. The proposed method can be used to estimate the mean weight of fish in aquaculture cages as an alternative to target strength measurements. Full-waveform acquisition and signal correlation techniques permitted to increase the signal-to-noise ratio and to improve the performance against traditional envelope-based echosounding.
\end{abstract}

Keywords: aquaculture, fish biomass, echosounder, waveform, pulse compression, fish morphometrics

\section{INTRODUCTION}

This work proposes an experimental acoustical method to estimate individual fish weight distribution of freely swimming fish, with special interest in its application to the monitoring of aquaculture floating cages. Fish target strength

5 (TS) determination is the basis for acoustical fish size estimation in acoustical fisheries stock assessment. [1, 2, 3, 4]. The TS of a scatterer is defined as the logarithmic expression of the ratio of the backscattered wave intensity at $1 \mathrm{~m}$

*email: vespinos@fis.upv.es

Preprint submitted to Aquacult. Eng.

March 29, 2017 
to the incident wave and it is evaluated in fisheries acoustics from the single echo envelope characteristics recorded with calibrated scientific echosounders. The error sources in near range TS measurements have been widely discussed in several works [5, 6, 7, 8] including near field effects, partial insonification of fish, relative size of fish to beam volume, and related point-source violations, time-varying-gain accuracy, orientation of fish, etc. All these issues have been specially considered in the application to fish growth monitoring in aquaculture of the referred work is the lack of correlation between TS and fish length with dorsal aspect measurements. This correlation is only possible from ventral aspect measurements, with the ultrasonic transducer placed below the cage or in the pen bottom. Under this unusual geometry, acoustical techniques are recognized as good candidates for biomass monitoring of caged fish. Our approach is addressed to obtain direct biometrics-related measurements, instead of a relationship between backscattered energy and length of the specimens, and to avoid the reported restriction to ventral aspect measurements.

Echo forms can provide information about the size, constitution and orientation of a sonar target, which has been investigated since the early stages of echo-sounding techniques [10]. Burwen and Fleischmann developed an echolength analysis approach in different references [11, 12, 13, 14] with a basic assumption: the bigger the fish the longer the resulting echo for a given emitted pulse duration and fish orientation. In those seminal works the echo length analysis was performed with acoustical data obtained with state-of-art scientific echosounders and enveloped-based echo analysis, to perform TS measurements of different fresh water fish species. Their results showed the possibility of performing fish size classification following the echo length criterion and even the capability of differentiating among species with similar TS ranges.

Improvements to the technological limitations of scientific echosounders were given by Stanton and different co-workers, with the introduction of broadband emitting and acquiring systems and of pulse-compression techniques to improve signal to noise ratio (SNR) and temporal (spatial) resolution of pulses [15, 16]. 
The improvement of spatial resolution allowed individual scattering features to be resolved within the fish. It also helped to observe that the time separations between the first and last returns of the compressed echoes were strongly correlated with the angle of orientation of fish for a wide range of angles. Using that information, they concluded that the orientation of individual fish can be inferred from the processed broadband echo from a single ping [17]. Moreover, pulse-compression of broadband echoes offered relevant information in the comparison of measured backscattering data, computed target strength simulations and provided insight about the dominant scattering mechanisms from fish inner structures [18].

The potential of cross-correlation techniques was also present in a different 50 line of work: fish counting or sizing in tanks. However in this case through the estimation of the so-called total target strength of a scatterer in a perfectly diffuse field [19], which involved the evaluation of the impulse response in a tank with moving fishes using different time and positions recordings.

The underlying idea in this work is to obtain a direct or indirect measurement of fish height from the time of flight (TOF) differences of echoes produced in the physical limits or internal structures of an individual fish, swimming perpendicularly to the acoustic beam axis. In principle there are no theoretical considerations that could limit the application of this technique measuring either from the sea surface (dorsal aspect of fish) or from below of the cage (ventral 60 aspect).

Biometric models for different species have been commonly derived in fisheries research relating fish total length to fish biomass. These biometric relationships are useful tools in fish stock assessment, in fish physiological condition or morphometric and genetic studies [20]. They are a fundamental part of the fish biomass estimations based in stereoscopic image measuring systems, widely used in aquaculture installations [21]. More specifically, some models have been used to increase the precision of estimates from simultaneous measurements of fish length and height [23], showing additionally that fish height varies isometrically with fish body thickness and weight [24]. We assumed as starting hypothesis 
weight. In the following sections we will first describe the experimental setup used to obtain the full waveform acoustical backscattered signals, as well as the processing methodology and interpretation to extract biometric information of aquacultured gilt-head sea breams (Sparus aurata, Linnaeus 1758). Secondly, ventral aspect of fish, the discussion and conclusions in terms of the applicability of the proposed method to the monitoring of fish farming.

\section{MATERIAL AND METHODS}

\subsection{Fish collection and biometric measurements}

We sampled manually a collection of 1019 specimens of sea bream with weights comprised between 18 and $690 \mathrm{~g}$, and tested different types of regressions to establish a weight $(\mathrm{W})$ to height $(\mathrm{H})$ relationship, to demonstrate the possibility of estimating gilt-head sea bream weight from height measurements in the most extended fish size range of production conditions.

Nevertheless, we limited our acoustical measurements to fish with heights that could be resolvable by TOF methods with the available configuration that will be introduced in the next subsection. We also wanted to study small differences in mean weight, just to insure that the resulting methods could be efficient growth monitoring tools for a better fish farm management. Therefore, from crements in mean weight between them were extracted. The average weights of each class were 159, 179, 194, 236 and $269 \mathrm{~g}$ with coefficient of variation lower than 0.2 , and fish heights between 6.5 and $8.5 \mathrm{~cm}$.

The experimental protocol was reviewed accordly with the Spanish Royal Decree 53/2013 on the protection of animals used for scientific purposes [29] and carried out out in accordance with The Code of Ethics of the World Medical Association (Declaration of Helsinki) for animal experiments. 


\subsection{Acoustical data acquisition and processing}

A small number of fish of each size class (4 individuals with exception of been treated and analysed with Matlab.

The fundamentals of TOF methods used in ultrasonic metrology or nondestructive testing establish that the spatial resolution is given by $c \tau / 2$, being $c$ the sound speed in the host medium and $\tau$ the pulse duration. The used water of approximately $4.5 \mathrm{~cm}$. The real one being even greater due to the transducer ringing time, and depending on detection threshold level. The use of a tone burst signal for pulse compression does not offer an improvement to 
the spatial resolution [25]. We performed a cross-correlation of the acquired time series with the excited signal in order to maximize the signal-to-noise-ratio (SNR) as indicated in [25, 26]. Once the signals were correlated, each ping envelope was calculated and sequentially represented in a classic echogram. From the echogram, single traces corresponding to individual targets were extracted, excluding all of those that could be the result of the superposition of fish trajectories at similar distances from the transducer. Also, the shape of the traces was considered, in order to restrict the study as much as possible to fish swimming horizontally (perpendicularly to beam axis). Therefore, only those with symmetrical form or with a limited slope in the echogram were selected. To estimate the real trajectory of fish was not possible since we used a single beam transducer and we did not know if the fish was crossing the beam center or not, and also swimming speed could not be obtained. Considering the longer traces as those originated when crossing close to the beam center and the average distance to the transducer, we estimated the trajectory slopes from trace slopes in the echogram and limited our data to those below 30 degrees. This was done in order to restrict the study as much as possible, only to fish swimming as horizontally as possible (perpendicularly to beam axis).

Each selected trace was individually analysed in terms of its ping-to-ping evolution. The characteristic profile of the echoes in each ping was studied, paying special attention to the repetitive patterns along the trace that could be directly related to the gilt-head sea bream biometric parameters. A preliminary analysis showed the existence of more than one local maximum in an elevated number of echoes, mainly in the central part of the traces corresponding to fish positions closer to the beam center and therefore less oblique. Traditionally, for TS measurement purposes, this situation has been avoided, increasing the emitted pulse length to link up single maxima echoes with individual fish.

Figure 1 (below-left) shows, as an example, the picture of three local maxima obtained for one of the targets of the biggest fish size class under study, revealing in principle the inner structure of the sea bream anatomy. We attributed the larger echo amplitude to the reflection from the fish swimbladder, and the previ- 

interfaces.

From the temporal difference between local maxima, information concerning fish height and anatomical distances should be obtained. Maximum attention should be paid to the algorithm used in the envelope detection for the analysed signals. The envelope of the correlated waveform was calculated and the maxima on it were the reference points for interval (distance) calculations.

The preliminary observation of the waveforms for the studied fish classes showed that most of the echoes exhibited the masking of the first water-tofish interface by the swimbladder (highest) maximum. Nevertheless, the third maximum was detectable in a major part of the central pings constituting the fish traces, as shown in Figure 3, a common feature of both ventral and dorsal measurements.

This common characteristic between measurements of both aspects (dorsal and ventral), with a bigger amplitude of the last maximum in comparison with the first one, when present, did not respond to an intuitive comprehension of the pulse propagation and echo formation and numerical simulations were performed in order to confirm our working hypothesis.

To simplify the analysis process (and to make an easier automation), the ping with largest amplitude among all of the pings making up the trace was established as a reference point. The full waveform for that ping was considered, and the temporal interval between the two latter local maxima provided the input for the distance calculation, assuming in a first approach a flesh sound speed equal to 1.025 times the sound speed of sea water during each measurement session[27].

The procedure was repeated for the previous and following pings of the trace, while the latter local maximum exceeded a certain measurable threshold.

The recordings obtained by means of fish ventral and dorsal insonifications were analysed with the above explained method and the results relating the obtained acoustical distances to fish weights are given in the following section. 

$\mathrm{cm}$ ) and the corresponding fish weights are shown. From the data we obtained a fitted fractional power expression of weight $(\mathrm{W})$ as a function of height $(\mathrm{H})$, 
$W=0.7536 H^{2.7791}$, with a correlation coefficient $R^{2}=0.9782$. This fitted curve Even in the cases that the echo maximum associated with the ventral interface 
was observed, its amplitude was always lower that the amplitude of the maximum corresponding to dorsal interface. Following the procedure explained in the Materials and Methods section, the distance between the maximum amplitude peak (attributed to the swimbladder interface) and the latter detected local maximum was determined, and a mean ventral acoustical height (VAH) was established for each group of sea breams as given in Table 1 .

Regarding the measurements obtained by means of dorsal insonification, the a Gumbel distribution model. Considering different weights, we obtained dif- 
ferent distributions but the same pattern. The different weights are associated with the same model of distribution but with different parameters depending on weight. In Tables 1 and 2, the sample size (n), the mode and the scale parameters of the fitted Gumbel model, the p-value for the Kolmogorov-Smirnov test, the theoretical means and standard deviations computed from the model are shown, considering the different weights for $D A H$ and $V A H$, respectively. Figure 5 shows the adjusted Gumbel-like models for the ventral case and the correlations of the model mean value $V A H_{G}$ with the sampled mean height $(H)$ and alternatively with the sampled mean length $(L)$ for each class. The adjustments are given by the following relations: $L=a \times 10^{b * V A H_{G}}$ with $a=4.0602$, $b=0.1326$ and $R^{2}=0.9927 ; H=a \times 10^{b * V A H_{G}}$ with $a=1.2024, b=0.1435$ and $R^{2}=0.9938$, with absolute errors below $1 \%$. Similar results are obtained for the dorsal case (not shown).

\section{DISCUSSION}

\subsection{Biometric relationships}

Fish weight for all growing stages of the collection of aquacultured gilthead sea breams can be predicted from fish height using the obtained biometric model of Figure 2. It should be noticed how fairly the prediction interval for the average fish weight (dashed lines) reproduces the variability of the real data and the high value of the regression coefficient for the minimum quadratic adjustment. Obviously, the obtained expression would depend on the particular genetics of the produced fish and their condition, as it is common to all biometric relationships, and should be tuned for each particular exploitation.

\subsection{Measured "acoustical heights"}

Echoes received by the transducer are originated from the reflections due to a change of impedance in the transmission medium. The larger echo amplitude can be attributed to the reflection from the fish swimbladder. In this case, the gas inside the bladder results in a high impedance change and therefore a 
great amount of energy is reflected to the transducer. Regarding the previous and later peaks, these should correspond respectively to the water-to-fish ("entrance") and fish-to-water ("exit") interfaces. The interface water-tissue implies a minor impedance change and accordingly provides less energy to the returned echo than the swimbladder. These phenomena are reflected in the difference of amplitudes among the maxima constituting the single fish echo.

The measurement of fish height by TOF techniques would be always limited by the spatial resolution of the acoustical setup. Fish vertical dimensions would depend on its size and its orientation, and the precision in the measurement of the water-to-fish and fish-to-water interfaces would be affected by the possible superimposition of the corresponding echoes with those produced by other inner structures. As it can be seen in the x-ray image of a gilt-head sea bream in Figure 1 (upper-left panel), the swimbladder is slightly tilted as it is common in several species, thus extending its contribution to the echo amplitude along the vertical direction.

The combination of the spatial resolution and the fish morphometrics allowed the measurement of distances related to biometric dimensions not considered initially, such as an averaged distance from swimbladder to the fish back (when measuring the ventral aspect) or to fish belly (from dorsal measurements).

325 The exact reproduction of the echo waveform characteristics in the experiment was not the objective of the performed numerical trials. Being cautious about the origin and result of the different backscattered waves superimpositions (e.g. head bone mixed with tissue boundaries), and aware of the differences with more realistic fish geometries, the simulations provided the necessary confidence to assume that the method allowed us to obtain an alternate acoustic height. The exponential expressions obtained for both VAH an-d DAH offered very good correlation cooeficients with fish weight, offering the VAH measurements a wider value interval for the measured weight collection. This fact supports the hypothesis that $V A H$ is related to biometrics of the upper part of the fish, whilst $D A H$ integrates information of the fish belly, since sea breams exhibit positive allometric growth [20], increasing faster the dorsal part of the fish than the 
ventral one. It can also be observed that the mean values adjust to the weight evolution in spite of being the standard deviations of the measured acoustical heights for each weight are quite high (up to 14 and $18 \%$ for dorsal and ventral

The p-values obtained for the Kolmogorov-Smirnov test, when analysing the statystical modelling of the measured acoustical heights are always much greater than 0.05, with the only exception of $V A H$ values associated with weight 194 g. The setting to a Gumbel distribution model is then statistically significant, and in most cases, the mean and the standard deviation of the adjusted models are similar to the mean and the standard deviation obtained from the samples, respectively. We must see the importance of the direct relationship of both measured acoustical heights to fish height (and also to fish length) as evidenced in Figure 5. This is an evidence of the interrelation of the different fish dimensions.

350 It must also be noted the low number of echoes needed to fit the probability distribution models, in clear contrast with target strength based methods [9] which would require much longer recording times.

\subsection{Applicability to production conditions and broadband techniques}

The approach presented here infers fish size for a given fish orientation (dorsal or ventral aspect) of a confined swimming fish. The variability in the fish orientation is directly projected into the dispersion of the measured acoustical heights and probably revealed by the different parametrization of the obtained Gumbel distributions for the different sizes. Nevertheless, their means arise as robust indicators of fish size. Fish orientations were determined in this experiment both by the beam aperture and the limited dimensions of the cage, that conditioned fish behaviour (with direct connection with their size), but also by the criteria of trace selection in the echogram (like in Figure 3).

Two questions arise when considering the applicability of the method to production conditions: first, the behaviour in a bigger cage of fish would produce a bigger dispersion of fish trajectories. Second, the associated high densities make difficult to obtain single fish tracks. Usually fish swim circularly in aquaculture 
cages, but they do it in a variety of trajectories not necessarily horizontal, occasionally performing vertical excursions. Our method extracts information about fish dimensions from selected fish traces in the echogram, those corresponding to fish swimming more closely to a perpendicular trajectory to the acoustic beam axis. In our experimental setup, the cage was small and the activity of fish reduced in comparison with production conditions, and therefore most of the tracks were horizontal, but we did select those traces from the echogram corresponding to the desired fish trajectories. The method can be generally applied to an echogram of production conditions taking into account that only symmetrical traces in the echogram correspond to horizontal fish trajectories and that those with limited slope in the echogram can correspond to limited slope in their physical trajectories. The fact that the acoustical fish height measurements fit a Gumbel distribution is an indication of the minimum condition of the measurable height if the fish is horizontal (perpendicular to beam axis), and the dispersion around that value is a consequence of the slight deviations from the desired fish orientation. Nevertheless the good fits obtained between manually sampled fish length and height versus the mean of the Gumbel fitted model for the acoustically measured height (Figure 5) assure good size estimations. The use of a split-beam system could add a stronger selection criterion by determining fish trajectory orientation and its relative position to beam axis, thus reducing dispersion in measurements.

The observation of individual fish traces has been a common problem in fisheries acoustics when estimating the biomass of a school and it was preferred to obtain in situ measurements of TS for such a purpose. The common method has been to obtain fish traces from fish on the periphery of schools or in less dense portions of school, these may not be representative of the total [28]. In any case this approach should be tested to evaluate its validity with farmed fish, selecting fish tracks from above or below the dense shoal. Transducer's beam aperture is another key point to consider on this question. A wider aperture (like the one used in the present work) would allow to insonify close fishes completely, above or below the school, and a narrower one could separate fish 
tracks at longer distances. A compromise on this subject should be explored in production conditions.

The spatial resolution of the employed technique is quite limited because of the use of narrow band pulses, being close to the distances to be resolved with the smallest fish sizes and species under study, but it could be easily improved using chirp signals. The resolution function of the emitted frequency sweep bandwidth $B W$ is then given by $c / 2 B W$, being $c$ the sound speed in the host medium. This is a new feature of the next generation of scientific echosounders (like Simrad-Kongsberg EK80), that can extend its application to all sizes of aquacultured fish, and therefore to any of the production cycles of commercial intensive exploitation in floating cages. It must be mentioned that the measurement of fish structures dimensions could also be used as an species differentiation variable for the development of complementary stock assessment algorithms in fisheries acoustics.

\section{CONCLUSIONS}

Full waveform analysis of fish echoes allowed us to obtain direct information of gilt-head sea bream morphometrics and to relate this information to fish weight. The measuring scheme (from sea surface or from cage bottom) does not have a practical influence on the viability of the fish weight estimation based on a TOF method. Future steps should now be taken both to the application of the presented technique in aquaculture production conditions, with higher fish densities, size and orientation distributions dispersions, as well as the extension of the technique to other aquacultured species.

\section{Acknowledgements}

This work was developed with the financial support of project ARM/1790/010 of the Tecnological Develoment Program of MAGRAMA, Spanish Government. E. Soliveres acknowledges support of Spanish Government grant AP2009-4459 FPU Subprogram. 
[1] K. J. Diercks and T. G. Goldsberry, Target Strength of a Single Fish, The Journal of the Acoustical Society of America, 48, 1B, pp.415-416, (1970)

[2] K. G. Foote, A. Aglen and O. Nakken, Measurement of fish target strength with a split beam echo sounder, The Journal of the Acoustical Society of America, 80,2, pp.612-621, (1986).

[3] K. G. Foote, Fish target strengths for use in echo integrator surveys, The Journal of the Acoustical Society of America, 87,3, pp. 981-987, (1987).

[4] J. Simmonds and D. N. Mac Lennan, Fisheries Acoustics-Theory and Practice, Blackwell Publishing, 2nd ed., (Oxford, England, 2005), 456 pp.

[5] D.N. MacLennan, Time varied gain functions for pulsed sonars, Journal of Sound and Vibration, 110, 3, pp. 511-522, (1986).

[6] M. Furusawa, M. Hamada, and C. Aoyama, Near range errors in sound scattering measurements of fish, Fisheries science, 65(1), pp. 109-116, (1999).

[7] J.J. Dawson, D. Wiggins, D. Degan, H. Geiger, D. Hart and B. Adams, Point-source violations: split-beam tracking of fish at close range, Aquatic Living Resources, 13, 05,9, pp. 291 - 295 (2000).

[8] M. Moszynski and A. Stepnowski,Increasing the Accuracy of Time-variedgain in Digital Echosounders, Acta Acustica united with Acustica, 88, pp. 814-817, (2002).

[9] F.R. Knudsen, J.E. Fosseidengen, F. Oppedal, O. Karlsen, and E. Ona, Hydroacoustic monitoring of fish in sea cages: target strength (TS) measurements on Atlantic salmon (Salmo salar), Fisheries Research, 69, 2, pp. 205 - 209, (2004).

[10] Hickling, R., Analysis of echoes from a solid elastic sphere in water, J. Acoust. Soc. Amer., 34 pp. 1582-1592, (1962). 
[11] D.L. Burwen, S. J. Fleischman, J.D. Miller and M. E. Jensen, Time-based signal characteristics as predictors of fish size and species for a side-looking hydroacoustic application in a river, ICES Journal of Marine Science: Journal du Conseil, 60, 3, pp. 662-668, (2003).

[12] D.L. Burwen, P.A. Nealson, S.J. Fleischman, T.J. Mulligan, and J.K. Horne, The complexity of narrowband echo envelopes as a function of fish side-aspect angle, ICES Journal of Marine Science: Journal du Conseil, 64, 5, pp. 1066-1074, (2007).

[13] S.J. Fleischman and D.L. Burwen, Mixture models for the species apportionment of hydroacoustic data, with echo-envelope length as the discriminatory variable, ICES Journal of Marine Science: Journal du Conseil, 60, 3, pp.592-598,(2003).

[14] S.J. Fleischman and D.L. Burwen, Correcting for position-related bias in estimates of the acoustic backscattering cross-section, Aquatic Living Resources, 13, 5, pp. 283 - 290, (2000).

[15] D. Chu and T.K. Stanton, Application of pulse compression techniques to broadband acoustic scattering by live individual zooplankton, J. Acoust. Soc. Am., 104, pp. 39-55, (1998).

[16] J.E Ehrenberg and T.C. Torkelson, FM slide (chirp) signals: a technique for significantly improving the signal-to-noise performance in hydroacoustic assessment systems, Fisheries Research, 47, pp. 193 - 199, (2000).

[17] T.K. Stanton, D.B. Reeder, and J.M. Jech, Inferring fish orientation from broadband-acoustic echoes, ICES J. Mar. Sci., 60, pp. 524-531, (2003).

475

[18] D.B. Reeder, J.M. Jech, and T.K. Stanton, Broadband acoustic backscatter and high-resolution morphology of fish: Measurement and modeling, J. Acoust. Soc. Am., 116, pp. 747-761, (2004). 
[19] S. G. Conti, P. Roux, C. Fauvel, B. D. Maurer, and D.A. Demer, Acoustical monitoring of fish density, behavior, and growth rate in a tank, Aquaculture, 251, pp. 314-323 (2006).

[20] G. Morey, J. Moranta, E. Massuti, A. Grau, M. Linde, F. Riera and B. Morales-Nin, Weight-length relationships of littoral to lower slope fishes from the western Mediterranean, Fisheries Research, 62, 1, pp. 89 - 96, (2003).

[21] M.R. Shortis, M. Ravanbakskh, F. Shaifat, E.S. Harvey, A. Mian, J.W Seager, P.F. Culverhouse, D.E. Cline and D.R. Edgington, A review of techniques for the identification and measurement of fish in underwater stereovideo image sequences, Proc. SPIE, 8791, pp. 87910G-87910G-10,(2013).

[22] Z. Boaz, The use of computer vision technologies in aquaculture A review, Computers and Electronics in Agriculture, 88,0, pp.125 - 132,(2012).

[23] R. E. Jones, R.J. Petrell, and D. Pauly, Using modified length-weight relationships to assess the condition of fish, Aquacult. Eng., 20, pp.261 - 276", (1999).

[24] H. Richter, C. Lueckstaedt, U. L. Focken and K. Becker, An improved procedure to assess fish condition on the basis of length-weight relationships, Arch. Fish. Mar. Res., 48, 3, pp. 226-235, (2000).

[25] M. A. Richards, Fundamentals of Radar Signal Processing, Second ed., McGraw-Hill Education, (New York, USA, 2014), Chapter 4, pp. 145-148, (688 pp.).

[26] M. Skolnik, Introduction to Radar Systems, McGraw Hill, (London, UK, 1980), Chapter 11, pp. 370-376, (640 pp.).

[27] N. Gorska, E. Ona, and R. Korneliussen, Acoustic backscattering by Atlantic mackerel as being representative of fish that lack a swimbladder. Backscattering by individual fish., ICES Journal of Marine Science, 62, pp.984-955, (2005). 
[28] R. J. Kloser, T. E. Ryan, G. J. Macaulay, and M. E. Lewis. In situ measurements of target strength with optical and model verification: a case study for blue grenadier, Macruronus novaezelandiae. ICES Journal of Marine Science, 68: 19861995 (2011).

[29] Boletín Oficial del Estado. Real Decreto 53/2013 sobre protección de animales utilizados en experimentación y otros fines científicos. BOE 2013; 34 (1): 1137011371 


\begin{tabular}{|c|c|c|c|c|c|c|c|c|}
\hline \multirow[b]{2}{*}{ Weight (g) } & \multicolumn{3}{|c|}{ Measurements } & \multicolumn{5}{|c|}{ Adjustment to Gumbel maxima distribution model } \\
\hline & Number of pings & Mean $\mathrm{VAH}(\mathrm{cm})$ & Standard & Mode & Scale & p-value & Model mean $(\mathrm{cm})$ & Standard deviation $(\mathrm{cm})$ \\
\hline & & & deviation $(\mathrm{cm})$ & & & & & \\
\hline 159 & 101 & 5.39 & 0.57 & 5.13491 & 0.432785 & 0.062605 & 5.38 & 0.56 \\
\hline 179 & 117 & 5.53 & 0.72 & 5.20275 & 0.569627 & 0.259701 & 5.53 & 0.73 \\
\hline 194 & 156 & 5.68 & 0.78 & 5.33222 & 0.561928 & 0.000204 & 5.66 & 0.72 \\
\hline 236 & 40 & 5.88 & 1.07 & 5.42115 & 0.728732 & 0.183354 & 5.84 & 0.93 \\
\hline 269 & 102 & 6.00 & 0.87 & 5.59955 & 0.647815 & 0.334027 & 5.97 & 0.83 \\
\hline
\end{tabular}

Table 1. Acoustical height measurements from ventral aspect (VAH) and its adjustment to a Gumbel-like distribution model. 


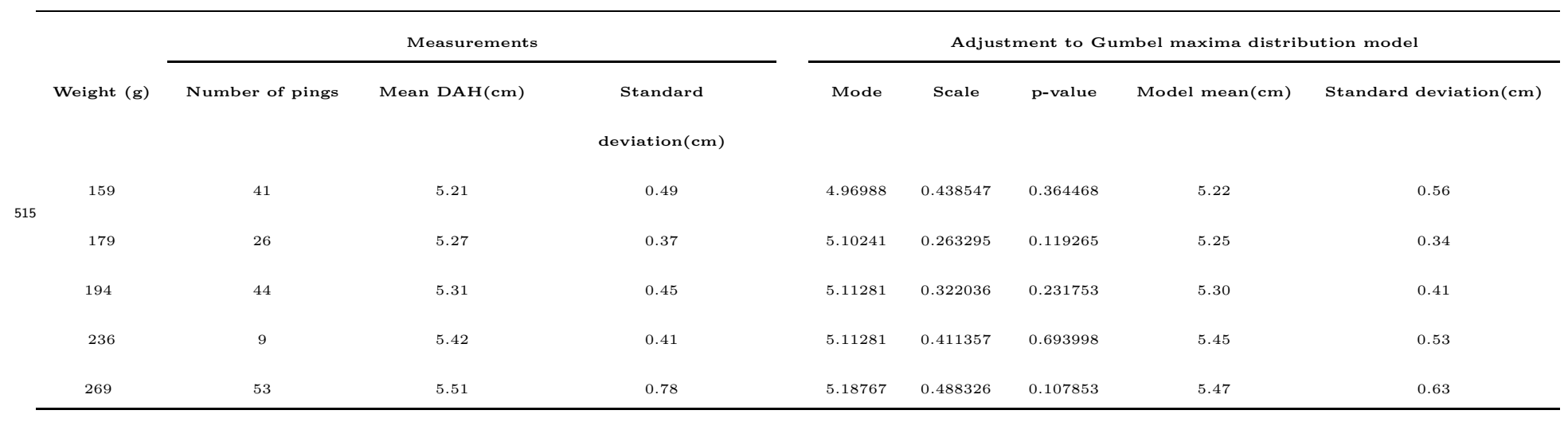

Table 2. Acoustical height measurements from dorsal aspect (DAH) and its adjustment to a Gumbel-like distribution model. 


\section{Figure Captions}

Figure 1. a)) Upper panel, left: x-ray image of a gilt-head sea bream weighting $330 \mathrm{~g}$. The swimbladder appears as a dark area below the spine. b) Lower

520 ping in a typical trace for the biggest sea bream size class measured in the experiment. c) Upper panel, right: Simplied geometry of a 3D axisymmetric sh used for numerical simulations. Semimajor axis of the ellipse corresponds to wave incidence direction. d) Lower panel, right: Correlated full waveform 525 obtained from a numerical simulation with the main simplied structures of gure c) with a 10 cycles incident pulse. Ventral incidence result is plotted both for experimental measurement and numerical simulation.

Figure 2. Sampled weight (W) versus height (H) plotting for 1019 aquacultured specimens of gilt-head sea bream (dots). The solid line represents the minimum quadratic adjustment of the data. The dashed line represents the $95 \%$ confidence interval for the average of the measured weights for the same fish height. The dotted line represents the $95 \%$ confidence interval for a new weight prediction for a given height.

Figure 3. An idealized representation of a fish trajectory and the correspond535 ing trace formation in the echogram as shown. We plot the obtained correlated waveforms for each ping, depending on its position in the selected trace.

Figure 4. Mean fish class weight versus both mean ventral and dorsal acoustical heights (labelled VAH and DAH, respectively).

Figure 5. a) Upper: Probability density functions (PDF) following a Gumbel540 like model of mean ventral acoustical heights for each size class. b) Lower: Linear representations for the correlation between the sampled mean length (height) of each size class and the mean obtained from the Gumbel model for the ventral acoustical height (VAH) distribution. 

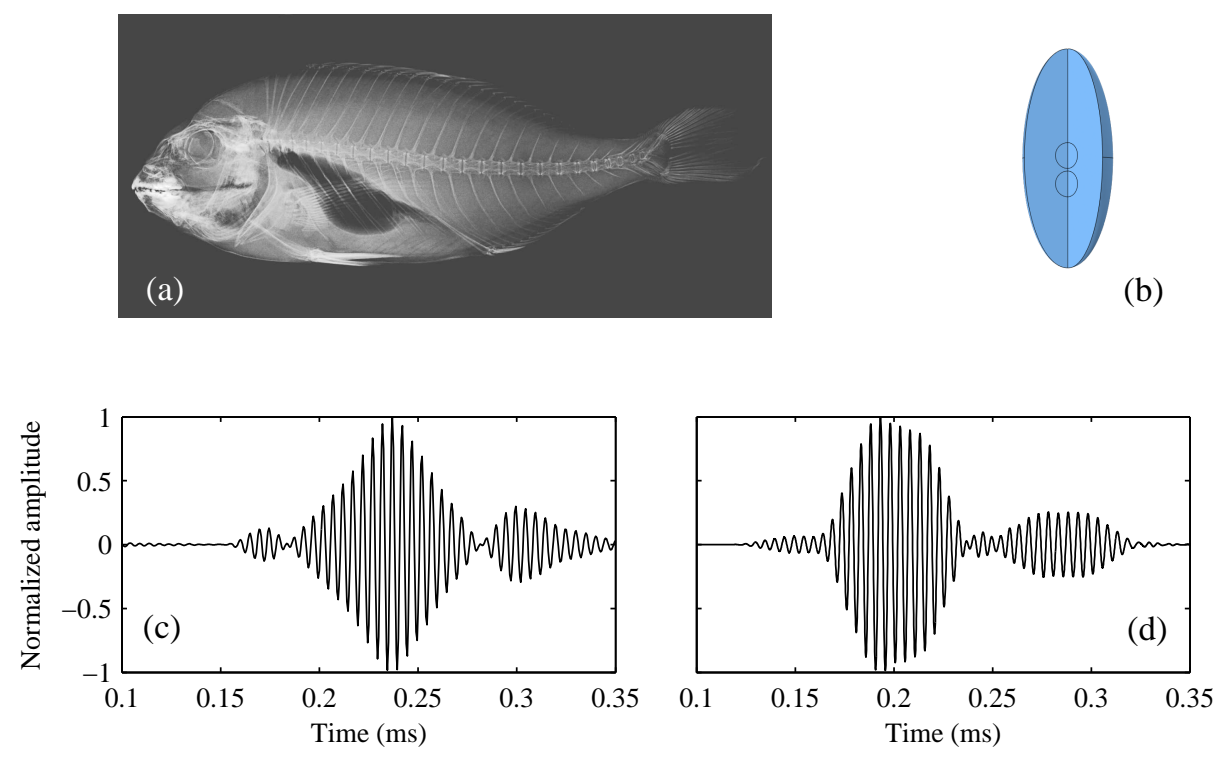
Figure 1. 


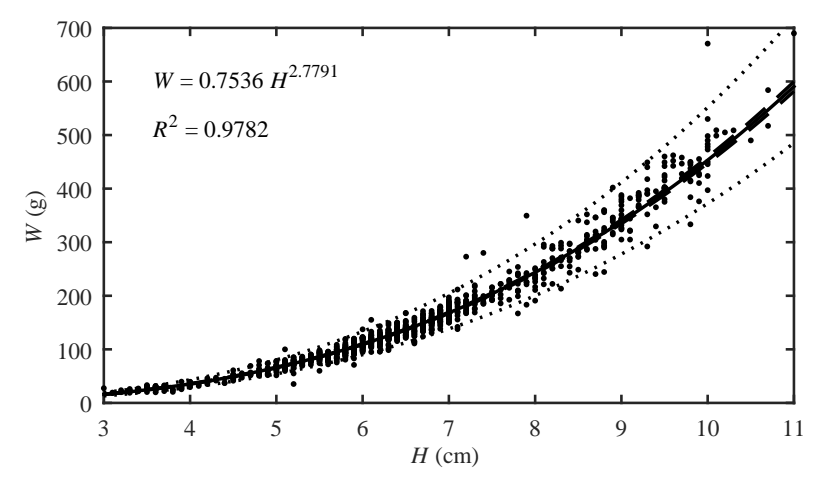

Figure 2 . 

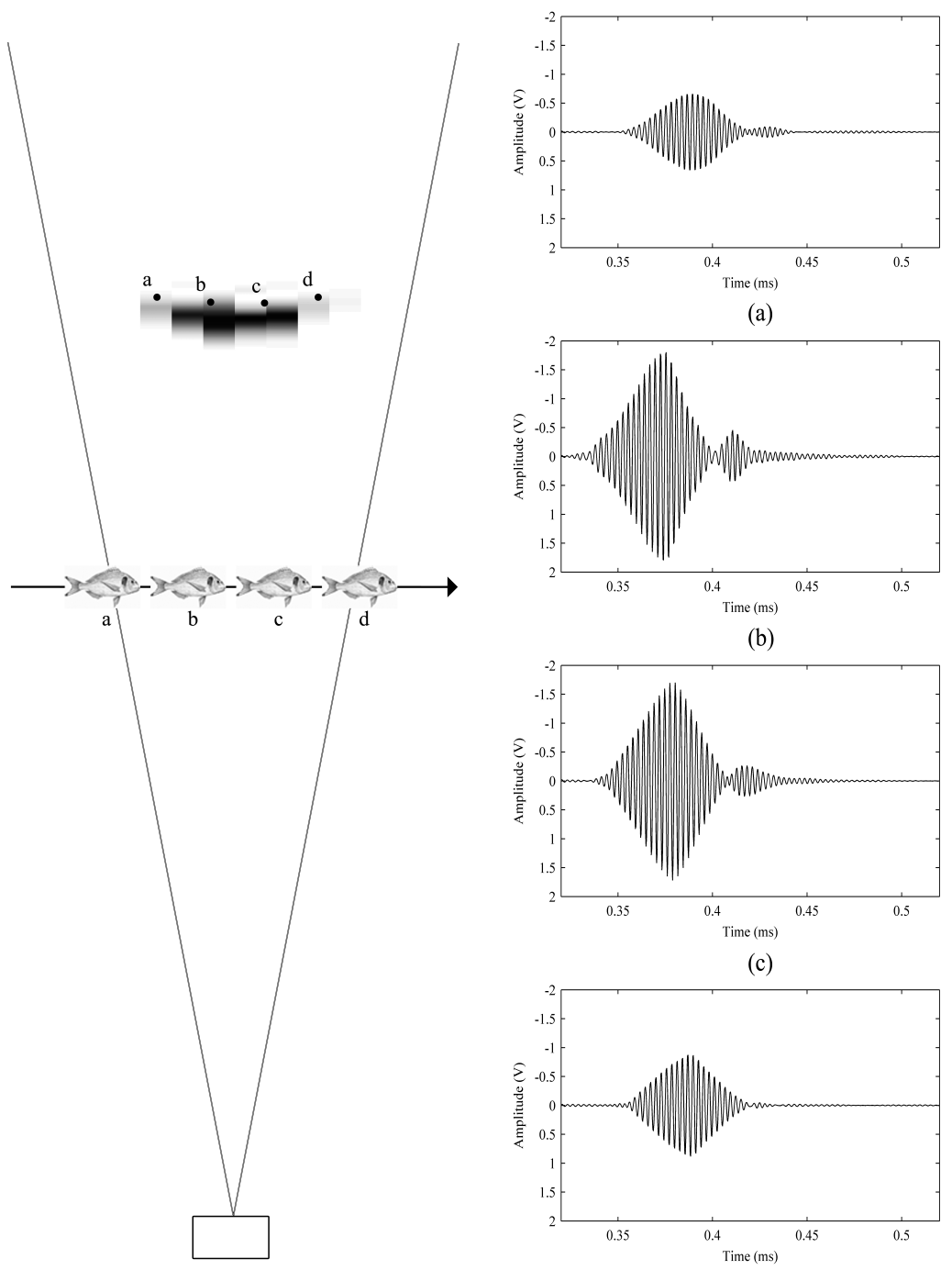

(a)

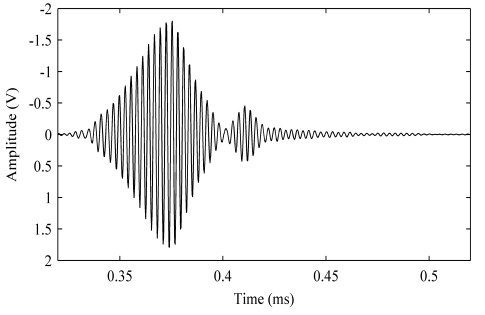

(b)

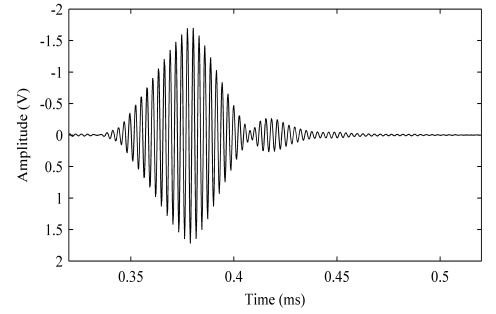

(c)

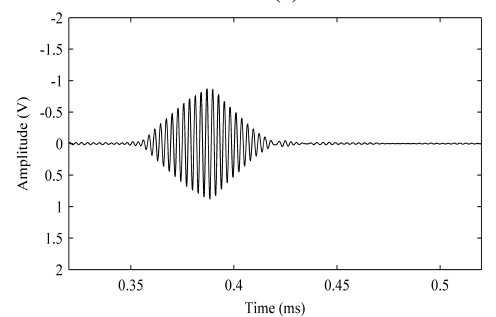

(d)

Figure 3. 


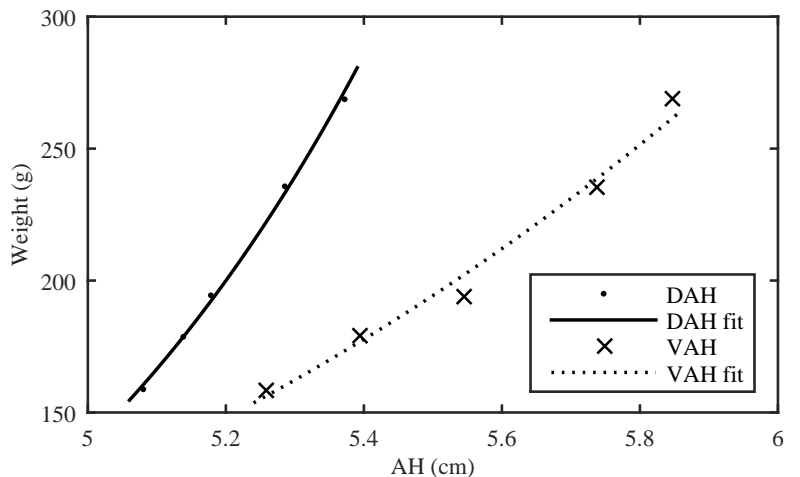

Figure 4 . 

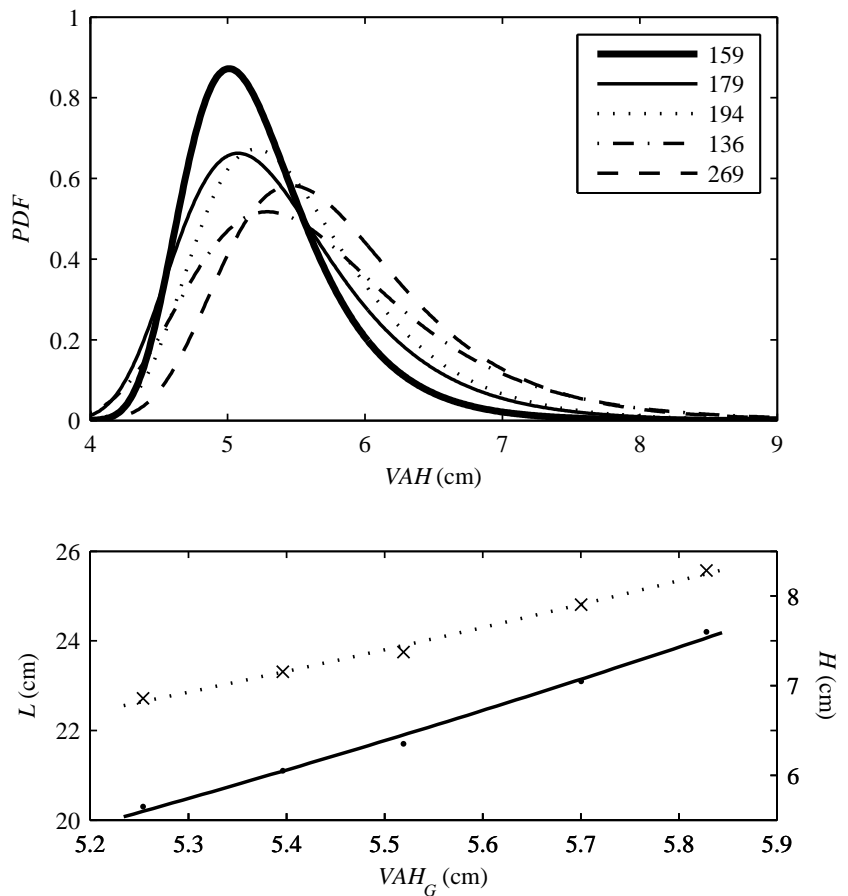

Figure 5. 